\title{
A New Class of Binary Zero Correlation Zone Sequence Sets
}

\author{
B. Fassi ${ }^{1}$, A. Djebbari ${ }^{1}$, Taleb-Ahmed. A ${ }^{2}$ And I. Dayoub ${ }^{3}$ \\ ${ }^{I}$ (Telecommunications and Digital Signal Processing Laboratory, Djillali Liabes University of Sidi Bel \\ Abbes, Algeria) \\ ${ }^{2}$ (Laboratoire LAMIH UMR, C.N.R.S, Université de Valenciennes et du Hainaut Cambresis, le Mont Houy, \\ France) \\ ${ }_{3}^{3}$ (I.E.M.N. Dept. O.A.E, U.M.R, C.N.R.S,Université de Valenciennes et du Hainaut CAMBRESIS, le Mont Houy, \\ France)
}

\begin{abstract}
This paper proposes a new class of binary zero correlation zone (ZCZ) sequence sets, in which the periodic correlation functions of the proposed sequence set is zero for the phase shifts within the zerocorrelation zone. It is shown that the proposed zero correlation zone sequence set can reach the upper bound on the ZCZ codes.
\end{abstract}

Keywords- Sequence design, theoretical upper bound, zero correlation zone (ZCZ) sequences.

\section{Introduction}

Zero correlation zone (ZCZ) sequences can be used in spread spectrum systems and CDMA systems to reduce the multiple access interference and co-channel interference [1]. There are several intensive studies on the constructing of ZCZ sequences set [1-8].

The ZCZ sequences set construction is limited by the correlation property and theory bounds [1-7]. Generally, sets of ZCZ sequences are characterized by the period of sequences L, the family size, namely the number of sequences $\mathrm{M}$, and the length of the zero-correlation zone $Z_{C Z}$. A $\mathrm{ZCZ}$ (L, $\mathrm{M}, Z_{C Z}$ ) sequence set that satisfies the theoretical bound defined by the ratio $M\left(Z_{c Z}+1\right) / L=1$ is called an optimal zero-correlation zone sequence set [4]. Compared with earlier works on binary ZCZ sequence sets [1,6], our proposed zerocorrelation zone sequence set is, in all cases, an optimal or an approach optimal ZCZ sequence set.

The paper is organized as follows. Section 2 introduces the notations required for the subsequent sections, the proposed scheme for sequence construction is explained in section 3. Examples of new ZCZ sequence sets are presented in Section 4. The properties of the proposed sequence sets are shown in Section 5. Finally, we draw the concluding remarks.

\section{Notations}

2.1 Definition 1: Suppose $X_{j}=\left(x_{j, 0}, x_{j, 1}, \ldots \ldots x_{j, L-1}\right)$ and, $X_{v}=\left(x_{v, 0}, x_{v, 1}, \ldots \ldots x_{v, L-1}\right)$ are two sequences of period $L$. Sequence pair $\left(X_{j}, X_{v}\right)$ is called a binary sequence pair if

$x_{j, i}, x_{v, i} \in\{-1,+1\}, i=0,1,2, \ldots \ldots \ldots L-1[7]$.

The Periodic Correlation Function (PCF) between $X_{j}$ and $X_{v}$ at a shift $\tau$ is defined by [4]:

$\forall \tau \geq 0, \theta_{\left(X_{j}, X_{v}\right)}(\tau)=\sum_{i=0}^{L-1} x_{j, i} x_{v,(i+\tau) \bmod (L)}$

2.2 Definition 2: A set of $M$ sequences $\left\{X_{0}, X_{1}, X_{2}, \ldots \ldots, X_{M-1}\right\}$ is denoted by $\left\{X_{j}\right\}_{j=0}^{M-1}$.

A set of sequences $\left\{\mathrm{X}_{\mathrm{j}}\right\}_{j=0}^{M-1}$ is called zero correlation zone sequence set, denoted by $\mathrm{Z}\left(\mathrm{L}, \mathrm{M}, \mathrm{Z}_{\mathrm{CZ}}\right)$ if the periodic correlation functions satisfy [4] :

$$
\begin{aligned}
& \forall j, 0<|\tau| \leq Z_{c z}, \theta_{\left(X_{j}, X_{j}\right)}(\tau)=0 \\
& \forall j,(j \neq v),|\tau| \leq Z_{c z}, \theta_{\left(X_{j}, X_{v}\right)}(\tau)=0
\end{aligned}
$$

\section{Proposed Sequence Construction}

In this section, a new method for constructing sets of binary ZCZ sequences is proposed.

The construction is accomplished through three steps.

3.1 Step 1: The $j^{\text {th }}$ row of the Hadamard matrix $H$ of order $n$ is denoted by

$h_{j}=\left[h_{j, 0}, h_{j, 1}, \ldots \ldots \ldots h_{j, n-1}\right]$. A set of $2 n$ sequences $d_{j}$, each of length $2 n$, is constructed as follows:

For $0 \leq j<n$, 
$d_{j+0}=\left[-h_{j}, h_{j}\right]$

$d_{j+1}=\left[h_{j}, h_{j}\right]$

3.2 Step 2: For a fixed integer value $n$, and for the first stage, $p=0$, we can generate, based on the scheme for sequence construction in [6], a series of sets $\left\{B_{j}\right\}_{j=0}^{2 n-1}$ of $2 n$ sequences as follows:

A sequence set $\left\{B_{j}\right\}_{j=0}^{2 n-1}$ is constructed from the sequences set $\left\{d_{j}\right\}_{j=0}^{2 n-1}$. A pair of sequences $B_{j+0}$ and $B_{j+1}$ of length $\left(2^{p+2} n\right)$ are constructed by the process of interleaving a sequence pair $d_{j+0}$ and $d_{j+1}$ as follows:

For $0 \leq j<n$,

$B_{j+0}=\left[d_{j+0,0}, d_{j+1,0}, d_{j+0,1}, d_{j+1,1}, \ldots \ldots, d_{j+0,2 n-1}, d_{j+1,2 n-1}\right]$

and,

$B_{j+1}=\left[d_{j+0,0},-d_{j+1,0}, d_{j+0,1},-d_{j+1,1}, \ldots \ldots ., d_{j+0,2 n-1},-d_{j+1,2 n-1}\right]$

The member size of the sequence set $\left\{B_{j}\right\}$ is $2 n$.

3.3 Step 3: For $p>0$, we can recursively construct a new series of set, $\left\{B_{j}\right\}_{j=0}^{2 n-1}$, by interleaving of actual $\left\{B_{j}\right\}_{j=0}^{2 n-1}$.

The $\left\{B_{j}\right\}_{j=0}^{2 n-1}$ is generated as follows:

For $0 \leq j<n$,

$B_{j+0}=\left[B_{j+0,0}, B_{j+1,0}, B_{j+0,1}, B_{j+1,1}, \ldots \ldots, B_{j+0,4 n-1}, B_{j+1,4 n-1}\right]$

and,

$B_{j+1}=\left[B_{j+0,0},-B_{j+1,0}, B_{j+0,1},-B_{j+1,1}, \ldots \ldots, B_{j+0,4 n-1},-B_{j+1,4 n-1}\right]$

The length of both sequences $B_{j+0}$ and $B_{j+1}$ is equal to $\left(2^{p+2} n\right)$.

\section{Example of Construction}

4.1 Step 1: Let $H$ be a Hadamard matrix of order $n=2$, given by:

$H=\left[\begin{array}{cc}1 & 1 \\ 1 & -1\end{array}\right]=\left[\begin{array}{l}h_{0} \\ h_{1}\end{array}\right]$

A set of $2 n=4$ sequences $d_{j}$, each of length $2 n=4$, is constructed as follows:

For $0 \leq j<2$,

$d_{0+0}=\left[-h_{0}, h_{0}\right]=[-1,-1,1,1]$

$d_{1+0}=\left[-h_{1}, h_{1}\right]=[-1,1,1,-1]$

$d_{0+1}=\left[h_{0}, h_{0}\right]=[1,1,1,1]$

$d_{1+1}=\left[h_{1}, h_{1}\right]=[1,-1,1,-1]$

4.2 Step 2: For the first iteration, $p=0$.

A pair of sequences $B_{j+0}$ and $B_{j+1}$ of length $\left(2^{p+2} n\right)=8$ are constructed by interleaving a sequence pair $d_{j+0}$ and $d_{j+1}$ as follows:

For $0 \leq j<2$,

$B_{j+0}=\left[d_{j+0,0}, d_{j+1,0}, d_{j+0,1}, d_{j+1,1}, \ldots \ldots, d_{j+0,3}, d_{j+1,3}\right]$

$B_{0+0}=[-1,1,-1,1,1,1,1,1]$

$B_{1+0}=[-1,1,1,-1,1,1,-1,-1]$

and,

$B_{j+1}=\left[d_{j+0,0},-d_{j+1,0}, d_{j+0,1},-d_{j+1,1}, \ldots \ldots, d_{j+0,3},-d_{j+1,3}\right]$

$B_{0+1}=[-1,-1,-1,-1,1,-1,1,-1]$

$B_{1+1}=[-1,-1,1,1,1,-1,-1,1]$

4.3 Step 3: For the next iteration $p=1$, The $\left\{B_{j}\right\}_{j=0}^{3}$ is generated as follows:

$B_{j+0}=\left[B_{j+0,0}, B_{j+1,0}, B_{j+0,1}, B_{j+1,1}, \ldots \ldots \ldots, B_{j+0,7}, B_{j+1,7}\right]$

$B_{0+0}=[-1,-1,1,-1,-1,-1,1,-1,1,1,1,-1,1,1,1,-1]$,

$B_{1+0}=[-1,-1,1,-1,1,1,-1,1,1,1,1,-1,-1,-1,-1,1]$,

and,

$B_{j+1}=\left[B_{j+0,0},-B_{j+1,0}, B_{j+0,1},-B_{j+1,1}, \ldots \ldots, B_{j+0,7},-B_{j+1,7}\right]$

$B_{0+1}=[-1,1,1,1,-1,1,1,1,1,-1,1,1,1,-1,1,1]$,

$B_{1+1}=[-1,1,1,1,1,-1,-1,-1,1,-1,1,1,-1,1,-1,-1]$. 
The length of both sequences $B_{j+0}$ and $B_{j+1}$ is equal to $\left(2^{p+2} n\right)=16$.

The member size of the sequence set $\left\{B_{j}\right\}$ is $2 n=4$.

Iteratively for $p=2$, The $\left\{B_{j}\right\}_{j=0}^{3}$ is generated as follows:

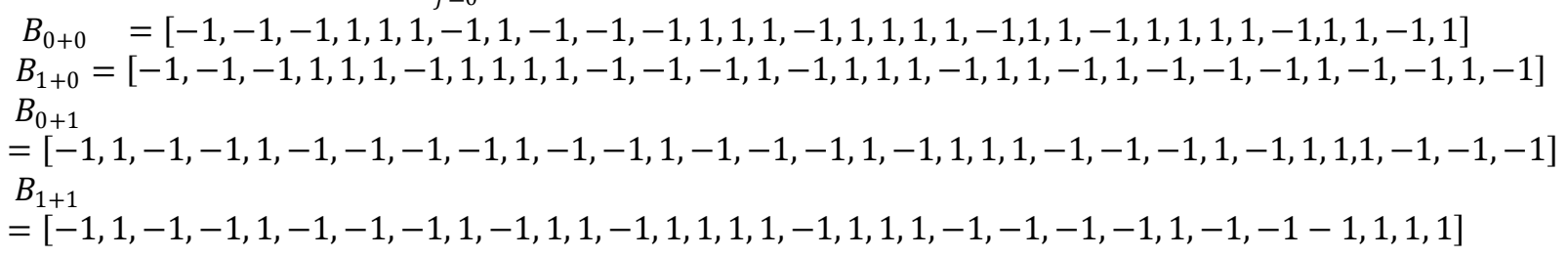

Also, we can find out that the zero correlation zone length of $\left\{B_{j}\right\}_{j=0}^{3}$ is 4 . For example, for $\tau=$ $0,1, \ldots \ldots \ldots 31$, the periodic auto-correlation functions (PACF) $(\forall j, j=v)$ given in (1) of $B_{0+0}, B_{1+0}, B_{0+1}$ and $B_{1+1}$ are calculated as follows:

$\theta_{\left(B_{0+0}, B_{0+0}\right)}(\tau)$

$=\{32,0,0,0,0,4,0,-4,16,-4,0,4,0,8,0,-8,0,-8,0,8,0,4,0,-4,16,-4,0,4,0,0,0,0\}$

$\theta_{\left(B_{1+0}, B_{1+0}\right)}(\tau)=$

$\{32,0,0,0,0,-4,0,4,-16,4,0,-4,0,8,0,-8,0,-8,0,8,0,-4,0,4,-16,4,0,-4,0,0,0,0\}$

$\theta_{\left(B_{0+1}, B_{0+1}\right)}(\tau)$

$=\{32,0,0,0,0,-4,0,4,16,4,0,-4,0,-8,0,8,0,8,0,-8,0,-4,0,4,16,4,0,-4,0,0,0,0\}$

$\theta_{\left(B_{1+1}, B_{1+1}\right)}(\tau)$

$=\{32,0,0,0,0,4,0,-4,-16,-4,0,4,0,-8,0,8,0,8,0,-8,0,4,0,-4,-16,-4,0,4,0,0,0,0\}$

The periodic cross-correlation functions (PCCF) $(\forall j, j \neq v)$ given in (1) are calculated as follows:

$\theta_{\left(B_{0+0}, B_{1+0}\right)}(\tau)$

$=\{0,0,0,0,0,-4,0,4,16,4,0,-4,0,0,0,0,0,0,0,0,0,4,0,-4,-16,-4,0,4,0,0,0,0\}$

$\theta_{\left(B_{0+0}, B_{0+1}\right)}(\tau)$

$=\{0,0,0,0,0,-4,-8,-4,0,4,-8,4,0,-8,-16,-8,0,8,-16,8,0,-4,-8,-4,0,4,-8,4,0,0,0,0\}$

$\theta_{\left(B_{0+0}, B_{1+1}\right)}(\tau)$

$=\{0,0,0,0,0,4,8,4,0,-4,8,-4,0,0,0,0,0,0,0,0,0,-4,-8,-4,0,4,-8,4,0,0,0,0\}$

$\theta_{\left(B_{1+0}, B_{0+1}\right)}(\tau)$

$=\{0,0,0,0,0,-4,-8,-4,0,4,-8,4,0,0,0,0,0,0,0,0,0,4,8,4,0,-4,8,-4,0,0,0,0\}$

$\theta_{\left(B_{1+0}, B_{1+1}\right)}(\tau)$

$=\{0,0,0,0,0,4,8,4,0,-4,8,-4,0,-8,-16,-8,0,8,-16,8,0,4,8,4,0,-4,8,-4,0,0,0,0\}$

$\theta_{\left(B_{0+1}, B_{1+1}\right)}(\tau)=\{0,0,0,0,0,4,0,-4,16,-4,0,4,0,0,0,0,0,0,0,0,0,-4,0,4,-16,4,0,-4,0,0,0,0\}$

We notice that the zero correlation zone length of $\left\{B_{j}\right\}_{j=0}^{3}$ is 4 .

The PACF and PCCF confirm that $\left\{B_{j}\right\}$ is a ZCZ $(32,4,4)$ sequence set.

\section{The Properties of the Proposed Sequence}

of order $n$

The proposed zero-correlation zone sequence set can be generated from an arbitrary Hadamard matrix

The length of $B_{j}$ in equations (6) and (7), equal to $\left(2^{p+2} n\right)$, is twice that of $B_{j}$ in equations (8) and (9).

The proposed ZCZ sequence set can satisfy the ideal autocorrelation and cross-correlation properties in the zero-correlation zone.

The generated sequence set satisfies the following properties:

$\forall j, \forall \tau \neq 0,|\tau| \leq 2^{p}$

$\theta_{\left(B_{j}, B_{j}\right)}(\tau)=0$

(10)

and,

$\forall j \neq v, \forall \tau,|\tau| \leq 2^{p}$

$\theta_{\left(B_{j}, B_{v}\right)}(\tau)=0$.

(11)

The $\left\{B_{j}\right\}$ is a ZCZ sequence set having parameters $\left(\mathrm{L}, \mathrm{M}, Z_{C Z}\right)=\left(2^{p+2} n, 2 n, 2^{p}\right)$, its parameters perfectly satisfy the theory bound of $\mathrm{ZCZ}$ sequences set $[4,7]$ : 
$M\left(Z_{C Z}+1\right) \leq L$

Let $\mu=\frac{M\left(Z_{C Z}+1\right)}{L}$, if $\mu=1$, it indicates that the ZCZ sequence set is optimal $[1,6]$.

For the proposed $Z C Z\left(2^{p+2} n, 2 n, 2^{p}\right)$ sequence set, $\mu=\frac{M\left(Z_{C Z}+1\right)}{L}=\frac{2 n\left(2^{p}+1\right)}{2^{p+2} n}=\frac{\left(2^{p}+1\right)}{2^{p+1}}$

1) For $p=0, \mu=1$, the proposed ZCZ sequence set is optimal.

2) For $p>0,1 / 2 \leq \mu<1$ and $\lim _{p \rightarrow \infty}(\mu)=1 / 2$.

For a given family size $\mathrm{M}$, we can construct different sets of sequences with different lengths $\mathrm{L}$ and zero correlation zone $Z_{C Z}$. As an example, assuming that $\mathrm{M}=16$, we can construct sets of ZCZ sequences with different lengths $\mathrm{L}=32,64,128,256,512,1024,2048,4096 \ldots$, and different $\mathrm{ZCZs}, Z_{C Z}=1,2,4,8,16,32,64$, $128, \ldots$ and different parameters $\mu=1,0.75,0.625,0.5625,0.53125,0.515625,0.5078125,0.5039063$,

In step 1 , other set of $2 n$ sequences $d_{j}$, each of length $2 n$, can be constructed as follows:

$1-$ For $0 \leq j<n$,

$d_{j+0}=\left[h_{j},-h_{j}\right]$

(13)

$d_{j+1}=\left[h_{j}, h_{j}\right]$

(14)

In this case, the set of $\left\{B_{j}\right\}_{j=0}^{3}$ is generated as follows:

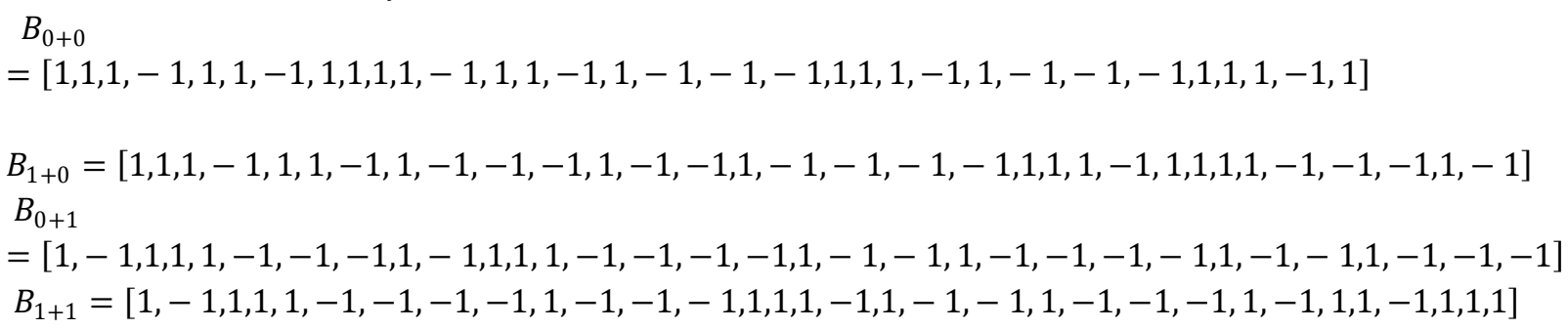

$2-$

$d_{j+0}=\left[h_{j}, h_{j}\right]$

For $0 \leq j<n$,

(15)

$d_{j+1}=\left[-h_{j}, h_{j}\right]$

(16)

The set of $\left\{B_{j}\right\}_{j=0}^{3}$ is generated as follows:

$$
\begin{aligned}
& B_{0+0}=[1,1,1,-1,-1,-1,1,-1,1,1,1,-1,-1,-1,1,-1,1,1,1,-1,1,1,-1,1,1,1,1,-1,1,1,-1,1] \\
& B_{1+0}= \\
& {[1,1,1,-1,-1,-1,1,-1,-1,-1,-1,1,1,1,-1,1,1,1,1,-1,1,1,-1,1,-1,-1,-1,1,-1,-1,1,-1]} \\
& B_{0+1}=[1,-1,1,1,-1,1,1,1,1,-1,1,1,-1,1,1,1,1,-1,1,1,1,-1,-1,-1,1,-1,1,11,-1,-1,-1] \\
& B_{1+1}=[1,-1,1,1,-1,1,1,1,-1,1,-1,-1,1,-1,-1,-1,1,-1,1,1,1,-1,-1,-1,-1,1,-1,-1,-1,1,1,1]
\end{aligned}
$$

3- $\quad$ For $0 \leq j<n$,

$d_{j+0}=\left[h_{j}, h_{j}\right]$

(17)

$d_{j+1}=\left[h_{j},-h_{j}\right]$

(18) 
It should be noted that in [9], the limits of the correlation function of binary zero-correlation zone sequences $\left\{B_{j}\right\}_{j=0}^{3}$ obtained from equation (17) and (18) are evaluated.

\section{Conclusion}

In this paper, we have proposed a new method for constructing sets of binary ZCZ sequences . The structure of the proposed ZCZ sequences set is simple and thus it is easy to be generated. The PACF side lobes and PCCF of the proposed sequence set is zero for the phase shifts within the zero-correlation zone. The proposed ZCZ sequence set with $\left(2^{p+2} n, 2 n, 2^{p}\right)$ is optimal or asymptotically optimal ZCZ sequence set. This method is useful for designing spreading sequences for multi-user CDMA system.

\section{References}

[1] P. Z. Fan, N. Suehiro, N. Kuroyanagi and X. M. Deng, Class of binary sequences with zero correlation zone, IEE Electronic Letters, 35(10), 1999, 777-779.

[2] H. Torii, M. Nakamura, N. Suehiro, A new class of zero-correlation zone sequence, IEEE. Trans. Inf. Theory, 50(3), 2004, 559-565.

[3] H. Torri, M. Nakamura, N. Suehiro, Enhancement of ZCZ sequence set construction procedure, Proc. IWSDA05, $2005,67-72$.

[4] T. Hayashi, A class of zero-correlation zone sequence set using a perfect sequence, IEEE Signal ProcessingLetters, 16(4),2009, 331334.

[5] Kai Liu, ChengqianXu Gang Li, Binary zero correlation zone sequence pair set constructed from difference set pairs, Proceedings of International Conference on Networks Security, Wireless Communications and Trusted Computing (NSWCTC'09),2, 2009, 543546.

[6] T. Maeda, S. Kanemoto, T. Hayashi, A Novel Class of Binary Zero-Correlation Zone Sequence Sets, $N^{\circ} 978-1-4244-6890-(02010$ IEEE, TENCON 2010.

[7] S. Renghui, Z. Xiaoqun, Li. Lizhi, Research on Construction Method of ZCZ Sequence Pairs Set, Journal of Convergence Information Technology, 6(1). January 2011.

[8] A. Rathinakumar and A.K. Chaturvedi, Mutually orthogonal sets of ZCZ sequences, ELECTRONICS LETTERS,40 (18),2004

[9] T. Hayashi, Limits of the Correlation Function of a Class of Binary Zero-correlation-zone Sequences, ftp://ftp.u-aizu.ac.jp/uaizu/doc/Tech-Report/2002/2002-1-013.pdf, June 6, 2002. 